\title{
Theory and Construction of Molecular Computers
}

\author{
Masami Hagiya \\ Graduate School of Science, University of Tokyo, \\ hagiya@is.s.u-tokyo.ac.jp, \\ Project home page: http://hagi.is.s.u-tokyo.ac.jp/MCP/
}

Molecular computing is a research area which aims to explore the potential of computation by molecules. Although the idea dates back to Feynman, it became realistic only when Adleman succeeded in solving a Hamiltonian path problem using DNA. The research community for the investigation and development of DNA Based Computers was then quickly formed by groups in the United States, Europe, Japan, etc.

The Japanese Molecular Computer Project, Theory and Construction of Molecular Computers, began in 1996 and will end in 2001. This project has produced a large number of experimental results, which verify the feasibility of basic operations for molecular computations and will help design a large scale molecular computer in the near future. In this, the last year of the project, a medium-scale DNA computer in which most reactions are executed by robot hands is under construction. A large number of theoretical results have also been produced by the project. Such theoretical studies either followed previous experimental results or initiated further experimental investigations.

I strongly believe that molecular computing should aim to explore the computational power inherent in molecular reactions. It should not be restricted to solving combinatorial problems by means of massive parallelism. In particular, a deep understanding of the computational power of molecules can be applied to many areas of information technology, biotechnology, and nanotechnology.

In this talk, after summarizing the achivements of our molecular computer project, I will suggest a set of perspectives to place the theory of molecular computing. This set can be roughly classified as follows.

- Studies which seek to propose or develop computational models suitable for describing molecular reactions. These include recent membrane models, which incorporate cell structure, in addition to simple molecular reactions.

- Studies on the computability of such computational models. In particular, achieving universal computability has been the major research interest in these studies. These include already classic ideas to build Turing machines using DNA.

- Studies on the complexity of such computational models. The trade-off between the number of computational steps and the amount of molecules necessary is a typical research issue. Note that molecular reactions should always be analyzed as parallel computations.

In addition to the above main stream of research, there are a large number of studies for analyzing the overall fidelity and efficiency of molecular computations. 
Related studies on encoding performance and design are also very active and important for reducing error and enhancing efficiency. Although the design of good encodings can be formulated as a combinatorial problem, it has recently been recognized that a thermodynamical treatment is essential for analyzing the error mechanisms inherent in molecular reactions.

According to statistical thermodynamics, the behavior of molecular reactions, and therefore that of molecular computations, is inherently probabilistic, even if the possibility of error is ignored. I believe that it is appropriate to analyze molecular computations as probabilistic processes, taking into account the physical properties of molecular reactions. Studies on the complexity of molecular computations should therefore include probabilistic analyses based on statistical thermodynamics. Such physical analyses will deepen our understanding of molecular reactions and will be applied to many areas as fundamental knowledge. 